\title{
A COMPARISON THEOREM FOR LINEAR DIFFERENCE EQUATIONS
}

\author{
P. W. ELOE
}

(Communicated by Kenneth R. Meyer)

\begin{abstract}
Let $a$ be real, $I=\{a, a+1, \cdots, b\}$ where $b-a$ is a positive integer or $I=\{a, a+1, \ldots\}$. Let $n$ be a positive integer and let $I^{n}=\{a, a+$ $1, \ldots, b+n\}$ if $b<\infty$ or $I^{n}=I$ otherwise. Consider the $n$th order difference equation $P u(m)=\sum_{j=0}^{n} \alpha_{j}(m) u(m+j)=0, \alpha_{n}(m)=1, \alpha_{0}(m) \neq 0$, $m \in I$. It is shown that if $0 \leq r(m) \leq q(m), m \in I$ and if the equations $P u(m)=0$ and $P u(m)+q(m) u(m)=0$ are disconjugate on $I^{n}$, then the equation $P u(m)+r(m) u(m)=0$ is disconjugate on $I^{n}$.
\end{abstract}

Let $a$ be real and let $I=\{a, a+1, \ldots, b\}$ where $b-a$ is a positive integer or $I=\{a, a+1, \ldots\}$. Let $n$ be a positive integer and define $I^{n}=\{a, a+1, \ldots, b+n\}$ if $b<\infty$ or $I^{n}=I$ otherwise. Consider the linear $n$th order difference equation

$$
P u(m)=\sum_{j=0}^{n} \alpha_{j}(m) u(m+j)=0,
$$

where $\alpha_{n}(m)=1, \alpha_{0}(m) \neq 0, m \in I$ and the coefficients $\alpha_{0}, \ldots, \alpha_{n}$ are defined on $I$. In a remark preceding Theorem 1, we shall indicate why the assumption that $\alpha_{0}(m)$ does not vanish for any $m \in I$ is made. The object of this paper is to show that if $P u(m)=0$ and $P u(m)+q(m) u(m)=0$ are disconjugate on $I^{n}$, where $0 \leq q(m)$ for $m \in I$, then if $0 \leq r(m) \leq q(m), m \in I$, the equation $P u(m)+r(m) u(m)=0$ is disconjugate on $I^{n}$. This is stated and proved in Theorem 4.

Oscillatory behavior of solutions and comparison theorems for difference equations have been of interest in recent years. For example, see $[1,3,4,6-8]$. Many of these authors have considered equations of the form

$$
P u(m)+q_{n-1}(m) u(m+n-1)+\cdots+q_{1}(m) u(m+1)=0 .
$$

Note that in these references the coefficient $q_{0}(m)$ for the lowest order term $u(m)$ is identically zero on $I$. In this paper, we adapt techniques employed by Nehari [5] for differential operators in order to handle the case $q_{0}(m) \geq 0$ on $I$.

We begin with the definition of the disconjugacy of (1) on $I^{n}$ and state criteria equivalent to the disconjugacy of (1) on $I^{n}$ due to Hartman [2]. For a finite or infinite sequence of real numbers $u=u(a), u(a+1), \ldots$, we say that $m=a$ is a generalized zero for $u$ if $u(a)=0$ and $m>a$ is a generalized zero for $u$ if $u(m)=0$ or

Received by the editors April 12, 1987. Presented at the 93rd Annual Meeting of the American Mathematical Society in San Antonio, January 21-24, 1987.

1980 Mathematics Subject Classification. Primary 39A10.

Key words and phrases. Disconjugacy, nonoscillation, comparison theorem, linear difference equation. 
there is an integer $k \geq 1$ such that $m-k \geq a,(-1)^{k} u(m-k) u(m)>0$ and if $k>1$, $u(m-k+1)=\cdots=u(m-1)=0$. The difference equation (1) is disconjugate on $I^{n}$ if no solution $u \not \equiv 0$ has $n$ generalized zeros on $I^{n}$.

Let $u_{1}, \ldots, u_{n}$ be $n$ real-valued functions defined on $I^{n}$. Let $k \in\{1, \ldots, n\}$, let $1 \leq r_{1}<\cdots<r_{k} \leq n$, and define

$$
W\left(r_{1}, \ldots, r_{k}\right)(m)=\operatorname{det}\left\{u_{r_{i}}(m+j)\right\}, \quad 1 \leq i, j \leq k .
$$

Let $\Delta^{0} u(m) \equiv u(m), \Delta u(m)=u(m+1)-u(m)$ and $\Delta^{j} u(m)=\Delta\left(\Delta^{j-1} u(m)\right)$, $j \geq 2$. Note that by determinant properties and elementary row operations

$$
W\left(r_{1}, \ldots, r_{k}\right)(m)=\operatorname{det}\left\{\Delta^{j-1} u_{r_{i}}(m)\right\}, \quad 1 \leq i, j \leq k .
$$

REMARK. Determinant identities are employed throughout this paper; in particular, nonzero determinants play a vital role in this development. It is here that we require $\alpha_{0}(m) \neq 0$ for any $m \in I$. For example, by (1) and (2) it readily follows that if $u_{1}, \ldots, u_{n}$ are $n$ solutions of $(1)$ on $I^{n}$ then $W(1, \ldots, n)(m+1)=$ $(-1)^{n} \alpha_{0}(m) W(1, \ldots, n)(m)$. Thus, a fundamental result concerning the solution set of $(1)$ is that $W(1, \ldots, n)(a) \neq 0$ if and only if $W(1, \ldots, n)(m) \neq 0$ for any $m \in I$. See Fort $[1]$ for further fundamental results concerning (1).

The following theorem is due to Hartman [2, Theorem 5.1].

THEOREM 1. The following are equivalent:

(i) (1) is disconjugate on $I^{n}$;

(ii) there exists a fundamental set of solutions $u_{1}, \ldots, u_{n}$ of (1) on $I^{n}$ such that $W(1, \ldots, k)(m)>0$ on $I^{n-k+1}, 1 \leq k \leq n$.

(iii) there exist positive functions $s_{k}(m), m \in I^{n-k+1}, 1 \leq k \leq n$, such that

$$
P u(m)=s_{n+1}(m) \Delta\left[s_{n}(m) \Delta\left[\cdots \Delta\left(s_{1}(m) u(m)\right) \cdots\right]\right], \quad m \in I,
$$

where $s_{n+1}=1 / s_{1} \cdots s_{n}$;

(iv) $u(m) \equiv 0$ is the only solution of (1) having $k(\geq 0)$ successive zeros at $m=a, \ldots, a+k-1$ and $n-k$ successive generalized zeros at $m=j, \ldots, j+n-k-1$, for some $j, a+k \leq j \in I^{k+1}$. (By $k=0$, we mean that $u(a) \neq 0$ if $a<j$.)

REMARK. In the proof of $f \Rightarrow a$ in Theorem 5.1, Hartman [2] proves a stronger statement than (iv). The stronger statement is that $u(m) \equiv 0$ is the only solution of (1) having $k(\geq 0)$ successive zeros at $m=a, \ldots, a+k-1$, and $n-k-1$ successive zeros at $m=j, \ldots, j+n-k-2$ and a generalized zero at $m=j+n-k-1$, for some $j, a+k \leq j \in I^{k+1}$. We shall use this stronger statement in the proof of Theorem 4.

We now introduce notation which is motivated by the factorization of $P$ in (4) and is analogous to the notation employed by Nehari [5]. Let $P u(m)=0$ be disconjugate on $I^{n}$ and let $s_{1}, \ldots, s_{n+1}$ be as in (4). Define quasi-difference operators $P_{k}$ by

$$
\begin{gathered}
P_{0} u(m)=s_{1}(m) u(m), \quad m \in I^{n}, \\
P_{k} u(m)=s_{k+1}(m)\left(\Delta P_{k-1} u(m)\right), \quad m \in I^{n-k}, 1 \leq k \leq n .
\end{gathered}
$$

Thus, $P_{n} u(m) \equiv P u(m)$. Let $u_{1}, \ldots, u_{n}$ be $n$ real-valued functions defined on $I^{n}$. Let $k \in\{1, \ldots, n\}$, let $1 \leq r_{1}<\cdots<r_{k} \leq n$, and let $0 \leq i_{1}<\cdots<i_{k} \leq n$. For $m \in I^{n-i_{k}}$, define $D\left(i_{1}, \ldots, i_{k} ; r_{1}, \ldots, r_{k}\right)(m)=\operatorname{det}\left\{P_{i_{j}} u_{r_{l}}(m)\right\}, 1 \leq l, j \leq k$. Note 
that by determinant properties and elementary row operations as in (3), it follows that

$$
D\left(0, \ldots, k-1 ; r_{1}, \ldots, r_{k}\right)(m)=\prod_{j=1}^{k} \prod_{l=0}^{k-j} s_{j}(m+l) W\left(r_{1}, \ldots, r_{k}\right)(m)
$$

$1 \leq k \leq n, m \in I^{n-k+1}$. Thus, it follows that if Theorem 1(iii) holds, then

$$
D\left(0, \ldots, k-1 ; r_{1}, \ldots, r_{k}\right)(m)>0
$$

if and only if $W\left(r_{1}, \ldots, r_{k}\right)(m)>0$.

The following lemma provides a discrete analogue to differentiating determinant functions. Let

$$
A_{k}=\{\sigma:\{1, \ldots, k\} \rightarrow\{0,1\}\}
$$

LEMMA 2. Let $k \in\{1, \ldots, n\}$, let $0 \leq i_{1}<\cdots<i_{k} \leq n-1,1 \leq r_{1}<\cdots<$ $r_{k} \leq n$, and let $m \in I^{n-i_{k}-1}$. Then

$$
\begin{aligned}
& D\left(i_{1}, \ldots, i_{k} ; r_{1}, \ldots, r_{k}\right)(m+1) \\
& \quad=\sum_{\sigma \in A_{k}}\left(\prod_{j: \sigma(j)=1} \frac{1}{s_{i_{j}+2}(m)}\right) D\left(i_{1}+\sigma(1), \ldots, i_{k}+\sigma(k) ; r_{1}, \ldots, r_{k}\right)(m) .
\end{aligned}
$$

Note that if $i_{j}+\sigma(j)=i_{j+1}+\sigma(j+1)$, then the corresponding determinant is zero.

PrOOF. The proof is by induction on $k$. For $k=1$,

$$
\begin{aligned}
D\left(i_{1} ; r_{1}\right)(m+1) & =P_{i_{1}} u_{r_{1}}(m+1)=P_{i_{1}} u_{r_{1}}(m+1)-P_{i_{1}} u_{r_{1}}(m)+P_{i_{1}} u_{r_{1}}(m) \\
& =\left(1 / s_{i_{1}+2}(m)\right) D\left(i_{1}+1 ; r_{1}\right)(m)+D\left(i_{1} ; r_{1}\right)(m) .
\end{aligned}
$$

Let $k>1$ and assume that (6) holds for each $1 \leq l \leq k-1,0 \leq i_{1}<\cdots<i_{l} \leq$ $n-1$, and $1 \leq r_{1}<\cdots<r_{l} \leq n$. Employ a cofactor expansion along the $k$ th row to obtain

$$
\begin{aligned}
& D\left(i_{1}, \ldots, i_{k} ; r_{1}, \ldots, r_{k}\right)(m+1) \\
&= P_{i_{k}} u_{r_{1}}(m+1) D\left(i_{1}, \ldots, i_{k-1} ; r_{2}, \ldots, r_{k}\right)(m+1) \\
&+\cdots+P_{i_{k}} u_{r_{k}}(m+1) D\left(i_{1}, \ldots, i_{k-1} ; r_{1}, \ldots, r_{k-1}\right)(m+1) \\
&=\left(1 / s_{i_{k}+2} P_{i_{k}+1} u_{r_{1}}(m)+P_{i_{k}} u_{r_{1}}(m)\right) D\left(i_{1}, \ldots, i_{k-1} ; r_{2}, \ldots, r_{k}\right)(m+1) \\
&+\cdots+\left(1 / s_{i_{k}+2} P_{i_{k}+1} u_{r_{k}}(m)+P_{i_{k}} u_{r_{k}}(m)\right) \\
& \times D\left(i_{1}, \ldots, i_{k-1} ; r_{1}, \ldots, r_{k-1}\right)(m+1) .
\end{aligned}
$$


By the induction assumption

$$
\begin{aligned}
& P_{i_{k}} u_{r_{1}}(m) D\left(i_{1}, \ldots, i_{k-1} ; r_{2}, \ldots, r_{k}\right)(m+1) \\
& +\cdots+P_{i_{k}} u_{r_{k}}(m) D\left(i_{1}, \ldots, i_{k-1} ; r_{1}, \ldots, r_{k-1}\right)(m+1) \\
& =P_{i_{k}} u_{r_{1}}(m)\left(\sum_{\sigma \in A_{k-1}}\left(\prod_{j: \sigma(j)=1} \frac{1}{s_{i_{j}+2}(m)}\right)\right. \\
& \left.\times D\left(i_{1}+\sigma(1), \ldots, i_{k-1}+\sigma(k-1) ; r_{2}, \ldots, r_{k}\right)(m)\right) \\
& +\cdots+P_{i_{k}} u_{r_{k}}(m)\left(\sum_{\sigma \in A_{k-1}}\left(\prod_{j: \sigma(j)=1} \frac{1}{s_{i_{j}+2}(m)}\right)\right. \\
& =\sum_{\sigma \in A_{k-1}}\left(\prod_{j: \sigma(j)=1} \frac{1}{s_{i_{j}+2}(m)}\right) \\
& \left.\left.\times i_{\left(i_{1}+\sigma(1), \ldots, i_{k-1}+\sigma(k-1), i_{k} ; r_{1}, \ldots, r_{k}\right)(m)}+\ldots, i_{k-1}+\sigma(k-1) ; r_{1}, \ldots, r_{k-1}\right)(m)\right)
\end{aligned}
$$

using a cofactor expansion along the $k$ th row. Similarly,

$$
\begin{aligned}
P_{i_{k}+1} & u_{r_{1}}(m) D\left(i_{1}, \ldots, i_{k-1} ; r_{2}, \ldots, r_{k}\right)(m+1) \\
& +\cdots+P_{i_{k}+1} u_{r_{k}}(m) D\left(i_{1}, \ldots, i_{k-1} ; r_{1}, \ldots, r_{k-1}\right)(m+1) \\
= & \sum_{\sigma \in A_{k-1}}\left(\prod_{j: \sigma(j)=1} \frac{1}{s_{i_{j}+2}(m)}\right) \\
& \times D\left(i_{1}+\sigma(1), \ldots, i_{k-1}+\sigma(k-1), i_{k}+1 ; r_{1}, \ldots, r_{k}\right)(m) .
\end{aligned}
$$

Thus, (6) follows from (7).

We now consider linear difference equations of the form

$$
P u(m)=\lambda \alpha(m) u(m),
$$

where $\alpha(m) \geq 0, m \in I$ and $\lambda=1$ or $\lambda=-1$.

LEMMA 3. Assume $P u(m)=0$ is disconjugate on $I^{n}$. Let $u_{1}, \ldots, u_{n}$ be the fundamental set of solutions of (8) on $I^{n}$ satisfying the initial conditions

$$
P_{j-1} u_{l}(a)=\delta_{j l}, \quad 1 \leq j \leq n, 1 \leq l \leq n .
$$

If $\lambda=-1$, then all even order minors of $D(0, \ldots, n-1 ; 1, \ldots, n)(m)$ are nonnegative and nondecreasing on their maximal intervals of existence. If $\lambda=1$, then all odd order minors are nonnegative and nondecreasing on their maximal intervals of existence.

PROOF. The proof is based on an argument of Nehari [5, Theorem 3.1], who credits Mikusinski [4] with the motivation of the argument. Let $0 \leq i_{1}<\cdots<$ $i_{k} \leq n-1$ and $1 \leq r_{1}<\cdots<r_{k} \leq n$ be given. If $i_{k}<n-1$,

$$
D\left(i_{1}, \ldots, i_{k} ; r_{1}, \ldots, r_{k}\right)(n+1)(m+1)
$$


is given by (6). If $i_{k}=n-1$,

(10)

$$
\begin{aligned}
& D\left(i_{1}, \ldots, i_{k-1}, n-1 ; r_{1}, \ldots, r_{k}\right)(m+1) \\
& \quad=\sum_{\sigma \in A_{k}}\left(\prod_{j: \sigma(j)=1} \frac{1}{s_{i_{j}+2}(m)}\right) D\left(i_{1}+\sigma(1), \ldots, n-1+\sigma(k) ; r_{1}, \ldots, r_{k}\right)(m) .
\end{aligned}
$$

If $\sigma(k)=1$, employ (8) to obtain

$$
\begin{aligned}
D\left(i_{1}\right. & \left.+\sigma(1), \ldots, i_{k-1}+\sigma(k-1), n ; r_{1}, \ldots, r_{k}\right)\left(r_{\imath}\right) \\
& =(-1)^{k-1} \lambda\left(\alpha(m) / s_{1}(m)\right) D\left(0, i_{1}+\sigma(1), \ldots, i_{k-1}+\sigma(k-1) ; r_{1}, \ldots, r_{k}\right)(m) .
\end{aligned}
$$

Thus, the relations (6) and (10) may be regarded as the first order system of difference equations $U(m+1)=A(m) U(m)$, where the components of $U(m)$ have the form $D\left(i_{1}, \ldots, i_{k} ; r_{1}, \ldots, r_{k}\right)(m)$ and $i_{1}, \ldots, i_{k}$ range over all integers satisfying $0 \leq i_{1}<\cdots<i_{k} \leq n-1$ and $r_{1}, \ldots, r_{k}$ are fixed and satisfy $1 \leq r_{1}<\cdots<r_{k} \leq n$. By (6), the diagonal elements of $A(m)$ are all 1 and by (9), the components of $\bar{U}(a)$ are either zeros or ones; in particular, the components of $U(a)$ are nonnegative. If $\lambda=-1$ and $k$ is even, each element of $A(m)$ is positive. Thus, each component of $U(m)$ is nonnegative and nondecreasing.

If $\lambda=1$ and $k$ is odd, the result follows similarly.

We now state and prove the main result of the paper. This is a discrete version of Nehari's Theorem 4.1 [5].

THEOREM 4. Let $0 \leq r(m) \leq q(m), m \in I$, and assume the equations $P u(m)=$ 0 and $P u(m)+q(m) u(m)=0$ are disconjugate on $I^{n}$. Then the equation $P u(m)+$ $r(m) u(m)=0$ is disconjugate on $I^{n}$.

PROOF. First consider the equation $P u(m)+r(m) u(m)=0$ in the form

$$
P u(m)=-r(m) u(m)
$$

and let $u_{1}, \ldots, u_{n}$ be the fundamental set of solutions of (11) satisfying the initial conditions (9). Then by Lemma 3 all even order minors of $D(0, \ldots, n-1 ; 1, \ldots, n)(m)$ are nonnegative and nondecreasing. By (9),

$$
D(0, \ldots, n-k-1 ; k+1, \ldots, n)(a+k)>0 .
$$

Thus, if $n-k$ is even, $D(0, \ldots, n-k-1 ; k+1, \ldots, n)(m)>0, m \geq a+k$, and so by $(5)$,

$$
W(k+1, \ldots, n)(m)>0, \quad m \geq a+k .
$$

Now consider the equation $P u(m)+r(m) u(m)=0$ in the form

$$
M u(m) \equiv P u(m)+q(m) u(m)=(q-r)(m) u(m) .
$$

$M u(m)=0$ is disconjugate on $I^{n}$, so there exist positive functions $\sigma_{1}, \ldots, \sigma_{n+1}$ such that $M$ has the factorization given by (4) where each $s_{j}$ is replaced by $\sigma_{j}$. Define quasi-difference operators $M_{k}$ as we did $P_{k}$ where each $s_{j}$ is replaced by $\sigma_{j}$. Let $\hat{u}_{1}, \ldots, \hat{u}_{n}$ be the fundamental set of solutions of (13) satisfying the initial conditions

$$
M_{j-1} \hat{u}_{l}(a)=\delta_{j l}, \quad 1 \leq j \leq n, 1 \leq l \leq n
$$


Define

$$
\hat{D}\left(i_{1}, \ldots, i_{k} ; r_{1}, \ldots, r_{k}\right)(m)=\operatorname{det}\left\{M_{i_{j}} \hat{u}_{r_{l}}(m)\right\}
$$

and

$$
\hat{W}\left(r_{1}, \ldots, r_{k}\right)(m)=\operatorname{det}\left\{\hat{u}_{r_{l}}(m+j)\right\} .
$$

Then by Lemma 3 with $\lambda=1$, all odd order minors of $\hat{D}(0, \ldots, n-1 ; 1, \ldots, n)(m)$ are nonnegative and nondecreasing; in particular, if $n-k$ is odd,

$$
\hat{W}(k+1, \ldots, n)(m)>0, \quad m \geq a+k .
$$

Assume $P u(m)+r(m) u(m)=0$ is not disconjugate on $I^{n}$. Then, from the remark following Theorem 1 , there is some $k \geq 0$ and some solution $u \not \equiv 0$ such that $u$ has $k$ successive zeros at $m=a, \ldots, a+k-1$, and $n-k-1$ successive zeros at $m=j, \ldots, j+n-k-2$ and a generalized zero at $m=j+n-k-1, a+k \leq j \in I^{k+1}$. We first show that $u(j+n-k-1) \neq 0$. Since $u(a)=\cdots=u(a+k-1)=0$, $u=C_{k+1} u_{k+1}+\cdots+C_{n} u_{n}=\hat{C}_{k+1} \hat{u}_{k+1}+\cdots+\hat{C}_{n} \hat{u}_{n}$ by (9) and (14). Now, again by (9) and (14), there exists a nontrivial solution of (1) satisfying the boundary conditions $u(a)=\cdots=u(a+k-1)=0, u(j)=\cdots=u(j+n-k-1)=0$ if and only if $W(k+1, \ldots, n)(j)=\hat{W}(k+1, \ldots, n)(j)=0$. If $u(j+n-k-1)=0$, $W(k+1, \ldots, n)(j)=\hat{W}(k+1, \ldots, n)(j)=0$ which contradicts (12) if $n-k$ is even and contradicts (15) if $n-k$ is odd.

Now, assume $u(j+n-k-1) \neq 0$. We can assume $j>a+k$. For suppose $j=a+k$ and $k \geq 1$. Then $u$ has $n-1$ successive zeros at $m=a, \ldots, a+n-2$, and $(-1)^{n-k} u(a+k-1) u(a+n-1)>0$. But, $u(a+k-1)=0$, which is a contradiction. If $j=a+k$ and $k=0$, then $u$ has $n-1$ successive zeros at $m=a, \ldots, a+n-2$ and $u(a+n-1) \neq 0$. Then $u$ does not have a generalized zero at $m=a+n-1$, which is again a contradiction.

Also note that $u(a+k) \neq 0$ for if $u(a+k)=0$, then $u$ has $k+1$ successive zeros at $m=a, \ldots, a+k$ and $n-(k+1)$ successive zeros at $m=j, \ldots, j+n-(k+1)-1$, $a+k+1 \leq j$. Then, as above $W(k+2, \ldots, n)(j)=0$ or $\hat{W}(k+2, \ldots, n)(j)=0$ which contradicts (12) or (15) depending on whether $n-(k+1)$ is even or odd. So, by (9) and (14), $C_{k+1} \neq 0$ and $\hat{C}_{k+1} \neq 0$. Let $n-k$ be even and assume without loss of generality that $C_{k+1}=1$. Then

$$
W(k+1, \ldots, n)(j-1)=W\left(u, u_{k+2}, \ldots, u_{n}\right)(j-1)=u(j-1) W(k+2, \ldots, n)(j)
$$

and

$$
\begin{aligned}
W(k+1, \ldots, n)(j) & =W\left(u, u_{k+2}, \ldots, u_{n}\right)(j) \\
& =(-1)^{n-k-1} u(j+n-k-1) W(k+2, \ldots, n)(j) .
\end{aligned}
$$

Thus, $(-1)^{n-k-1} u(j-1) u(j+n-k-1)>0$. But $u$ has a generalized zero at $j+n-k-1$ and so, $(-1)^{n-k} u(j-1) u(j+n-k-1)>0$. Thus, $n-k$ is not even.

By a similar argument, assuming $\hat{C}_{k+1}=1$, it follows that $n-k$ is not odd. Thus, $P u(m)+r(m) u(m)=0$ is disconjugate on $I^{n}$ and the proof is complete.

\section{REFERENCES}

1. T. Fort, Finite differences, Oxford Univ. Press, Oxford, 1948.

2. P. Hartman, Difference equations: disconjugacy, principle solutions, Green's functions, complete monotonicity, Trans. Amer. Math. Soc. 246 (1978), 1-30. 
3. J. W. Hooker and W. T. Patula, Riccati type transformations for second-order linear difference equations, J. Math. Anal. Appl. 829 (1981), 451-462.

4. J. Mikusinski, Sur l'equation $x^{(n)}+A(t) x=0$, Ann. Polon. Math. 1 (1955), 207-221.

5. Z. Nehari, Disconjugate linear differential operators, Trans. Amer. Math. Soc. 129 (1967), $500-516$.

6. W. T. Patula, Growth, oscillation and comparison theorems for second order linear difference equations, SIAM J. Math. Anal. 10 (1979), 1272-1279.

7. A. C. Peterson, Existence and uniqueness theorems for nonlinear difference equations, J. Math. Anal. Appl. 125 (1987), 185-191.

8. B. Smith and W. E. Taylor, Jr., Oscillatory and asymptotic behavior of certain fourth order difference equations, Rocky Mountain J. Math. 16 (1986), 403-406.

Department of Mathematics, University of Dayton, Dayton, Ohio 45469 